\title{
Are higher blood flow restriction pressures more beneficial when lower loads are used?
}

\author{
SJ Dankel, MB Jessee, SL Buckner, JG Mouser, KT Mattocks, JP Loenneke
}

Department of Health, Exercise Science, and Recreation Management, Kevser Ermin Applied Physiology Laboratory, The University of Mississippi, University, MS, USA

Received: December 20, 2016

Accepted: June 22, 2017

\begin{abstract}
The application of blood flow restriction during low-load resistance exercise has been shown to induce muscle growth with high or low restriction pressures, however, loads lower than $20 \%$ one-repetition maximum (1RM) remain unexplored. Fourteen trained individuals completed six elbow flexion protocols involving three different loads $(10 \%, 15 \%$, and $20 \% 1 \mathrm{RM})$ each of which was performed with either a low ( $40 \%$ arterial occlusion) or high (80\% arterial occlusion) pressure. Pre- and post-measurements of surface electromyography (sEMG), isometric torque, and muscle thickness were analyzed. An interaction was present for torque $(p<0.001)$ and muscle thickness $(p<0.001)$ illustrating that all increases in pressure and/or load resulted in a greater fatigue and muscle thickness. There was no interaction for sEMG $(p=0.832)$; however, there were main effects of condition $(p=0.002)$ and time ( $p=0.019)$ illustrating greater sEMG in the $20 \% 1 \mathrm{RM}$ conditions. Higher blood flow restriction pressures may be more beneficial for muscle growth when very low loads are used.
\end{abstract}

Keywords: electromyography, elbow flexion, occlusion training, resistance training, strength training, hypertrophy, swelling

\section{Introduction}

When combined with low-load resistance exercise, the application of blood flow restriction has been shown to increase muscle size and strength of the upper (6) and lower body (22), and this has been attributed to a number of potential mechanisms [for reviews, see $(21,25)$ ]. While the majority of studies using blood flow restriction in combination with low-load resistance exercise use loads corresponding to $20 \%$ or $30 \%$ of an individual's one-repetition maximum (1RM) $(6,22)$, no studies to our knowledge have performed isotonic exercise using loads even lower than 20\% 1RM. One study found that isotonic training with $15 \%$ of maximal voluntary isometric contraction (MVC) strength increased muscle size (14), but it is unknown what percentage of 1RM this corresponds to. Similarly, applying blood flow restriction during bodyweight exercises (i.e., lunges and squats) has demonstrated small (15) to no improvements (11) over exercise in the absence of blood flow restriction, but it is once again unknown what percentage of 1RM these bodyweight exercises correspond to or exactly what pressure was applied. Other low-intensity activities, such as walking (2) and cycling (1), have been shown to increase muscle size, illustrating that more studies are needed to

Corresponding author: Jeremy P. Loenneke, PhD

Department of Health, Exercise Science, and Recreation Management, Kevser Ermin Applied Physiology Laboratory, The University of Mississippi

231 Turner Center, University, MS 38677, USA

Phone: +1 662915 5567; Fax: +1 662915 5525; E-mail: jploenne@olemiss.edu 
examine the efficacy of blood flow restriction when combined with even lower loads (i.e., $<20 \% 1 \mathrm{RM}$ ).

Performing low-load exercise to volitional failure has been shown to increase muscle size similar to that of blood flow-restricted exercise, albeit requiring a greater number of repetitions at the same relative load $(8,9)$. While low-load exercise to volitional failure increases muscle size, there is likely a minimal loading threshold required to induce volitional failure through reductions in blood flow via contraction-induced increases in intramuscular pressure (13). Thus, artificially reducing blood flow through the application of blood flow restriction may be of greater importance when lower loads are used, with higher pressures creating an anabolic environment by inducing a greater level of fatigue (5). The importance of the blood flow restriction pressure has been examined in conjunction with $20 \%$ and $30 \%$ $1 \mathrm{RM}$ loads demonstrating that sEMG activity peaked at around $40 \%-50 \%$ of arterial occlusion pressure $(5,20)$, but lower loads may benefit from even higher pressures. The idea that higher pressures may be more advantageous when lower loads are used is supported by a training study detailing that increasing the pressure from $40 \%$ to $80 \%$ of arterial occlusion augmented muscle growth when used with a 20\% 1RM load but had no greater effect when a $40 \%$ load was used (17). Furthermore, the application of blood flow restriction did not augment muscle growth, when applied during moderate- to high-load training using loads corresponding to $60 \%$ or $80 \%$ of $1 \mathrm{RM}(16)$.

The purpose of this study was to determine whether higher pressures augment acute muscular responses when exercising with very low exercise loads ( $\leq 20 \% 1 \mathrm{RM})$. Given the importance of effort (i.e., approaching or reaching volitional failure) and motor unit recruitment on inducing muscle growth (23), combined with the relationship between acute swelling and muscle hypertrophy $(18,29)$, we sought to investigate the acute changes in sEMG, swelling, and torque immediately following each bout of blood flow-restricted exercise. We hypothesized that increasing the pressure and/or load would result in higher sEMG, greater torque decrements, and greater acute swelling.

\section{Materials and Methods}

\section{Participants}

A total of 14 resistance-trained (regularly performing upper-body resistance exercises, which included elbow flexion exercise twice per week) individuals (11 males and 3 females) between the ages of 18 and 35 were recruited for the study. Resistance-trained individuals were chosen so that the elbow flexion exercise was not a novel stimulus and allowed strength levels to remain stable across time. All individuals provided informed written consent for this study, which was approved by the University's Institutional Review Board. For all visits, individuals were instructed to refrain from (1) eating within $2 \mathrm{~h}$, (2) consuming caffeine within $8 \mathrm{~h}$, and (3) exercising within $24 \mathrm{~h}$. Participants were excluded from the study, if they were tobacco users or had one or more predisposing risk factors for thromboembolism (24).

\section{Study design}

Participants came to the laboratory on four separate days, at the same time of day, with each visit separated by 5-10 days. On visit 1, the familiarization with exercise consisted of 15 repetitions completed on each arm with a pressure corresponding to $50 \%$ of the individuals predetermined arterial occlusion pressure. This pressure was chosen to give individuals an 
idea of the exercise protocol without actually experiencing one of the included pressures. Visits 2-4 all involved the same procedures but differed based on the load lifted and level of restriction applied during exercise. Each of the exercise measurements began with a measure of arterial occlusion. Measurements of muscle size and MVC strength were taken before and immediately after the bout of exercise, while sEMG was taken during the preexercise MVC and throughout the exercise. Individuals then rested in the seated position for $15 \mathrm{~min}$ before performing the same procedure on the contralateral arm. Both arms were exercised for two conditions per session and thus six total conditions were completed over the three exercise sessions.

\section{Arterial occlusion pressure}

The arterial occlusion pressure was taken in the standing position and recorded as the minimal pressure in which a pulse was no longer present at the radial artery using an MD6 Doppler Probe (Hokanson, Bellevue, WA, USA). An E20 rapid cuff inflator (Hokanson) was used to inflate a 5-cm-wide nylon cuff (Hokanson) to $50 \mathrm{mmHg}$ before being progressively increased by $1 \mathrm{mmHg}$ increments until a pulse was no longer detected. This measurement was taken each visit following 10 min of quiet rest, except when taken between arms, in which case individuals were allotted $15 \mathrm{~min}$ of rest.

\section{$1 R M$}

A $1 \mathrm{RM}$ for the unilateral elbow flexor exercise was obtained on both arms for each individual on visit 1. Briefly, participants warmed up with a load corresponding to an estimated $30 \% 1$ RM. Following the brief warm-up, the load was increased to approximately $90 \%$ of the individuals 1RM and participants performed one repetition. Thereafter, the load was either increased or decreased until a 1RM was obtained. The dumbbell was handed to each individual at full elbow extension and participants were instructed to keep their back and heels against the wall during all 1RM attempts to ensure strict form. A 1RM was usually obtained between 3 and 5 repetitions.

\section{Muscle thickness}

Muscle thickness was measured at $70 \%$ of the distance between the acromion process and lateral epicondyle of the humerus. Using B-mode ultrasound (General Electric, Fairfield, CT, USA), the distance between the muscle-fat and muscle-bone interface was measured by placing a $10-\mathrm{MHz}$ linear array probe on the anterior portion of the upper arm while using conductive gel to avoid depressing the skin. Two images were then taken at each time point and the average of the two measurements was recorded as the muscle thickness. The coefficient of variation for this measurement was calculated as $2 \%$ and the minimal difference was calculated as $0.2 \mathrm{~cm}$ using methods described previously (26).

\section{Surface electromyography (sEMG)}

sEMG was recorded from the elbow flexors of each arm during exercise. Electrodes were placed between the medial acromion and the antecubital fossa at one third the distance from the antecubital fossa. The skin was shaved, abraded, and cleaned with alcohol wipes. Bipolar electrodes were placed over the muscle belly with an interelectrode distance of $20 \mathrm{~mm}$. The ground electrode was placed on the 7th cervical vertebrae at the neck (12). The surface electrodes were connected to an amplifier and digitized (iWorx, Dover, NH, USA). The signal was filtered (low-pass filter $500 \mathrm{~Hz}$; high-pass filter $10 \mathrm{~Hz}$ ), amplified 
$(1,000 \times)$, and sampled at a rate of $1 \mathrm{KHz}$. Before the exercise bout, the participant performed two MVCs on an isokinetic dynamometer (Biodex System 4 Medical Systems, Shirley, NY, USA) with the elbow flexors at a joint angle of $60^{\circ}$ with $60 \mathrm{~s}$ of rest between each attempt. sEMG was continuously recorded from the elbow flexors during each exercise bout. Computer software program (iWorx) was used to analyze the data. sEMG amplitude (root mean square) was analyzed from the average of the first 3 repetitions and the average of the last 3 repetitions for each set and expressed relative to the highest preexercise MVC (\%MVC).

\section{$M V C$}

Participants were asked to sit in an isokinetic dynamometer, with the seat and lever arm adjusted for each individual. After weighing the limb to correct for gravity, the lever arm was locked into place at $60^{\circ}$ of elbow flexion. Participants were then asked to contract using their elbow flexors by pulling against the lever arm as hard as possible for a 3-s period. Participants performed two contractions separated by a 1-min rest period on each arm, with the exception of the immediate postexercise MVC in which only one contraction was completed to avoid allowing additional recovery time. The maximum amount of torque produced was recorded as the MVC for that particular time point. Participants were blinded to their MVC results during testing. The coefficient of variation for this measurement was calculated as $7 \%$ and the minimal difference was calculated as $10.8 \mathrm{Nm}$ using methods described previously (26).

\section{Exercise protocol}

Unilateral elbow flexion exercise was performed using dumbbells. During each visit, participants were randomly assigned a load of either $10 \%, 15 \%$, or $20 \%$ of their $1 \mathrm{RM}$ and a pressure corresponding to either $40 \%$ or $80 \%$ of their predetermined arterial occlusion pressure (six total conditions). These specific pressures were chosen based on a previous study examining chronic muscle adaptations (17). Individuals then performed four sets of exercise consisting of 30 goal repetitions on the first set, and 15 goal repetitions for each of the following three sets. A 30-s rest period was given between each set. If individuals could not complete all of the goal repetitions, they stopped the exercise and rested until the start of the next set. This protocol was chosen to enable comparisons with previous studies $(5,20)$.

\section{Statistical analysis}

All statistical analyses were computed using SPSS version 23. A $6 \times 4$ repeated measures analysis of variance (ANOVA) was used to determine differences in repetitions across sets and sEMG during the first 3 and last 3 repetitions of each set. If there was an interaction, a one-way repeated measures ANOVA was performed within each condition across each set and within sets across each condition. If no interaction was present, main effects were analyzed. A $6 \times 2$ repeated measures ANOVA was used to determine differences in MVC strength and muscle thickness. If there was an interaction, a oneway repeated measures ANOVA was performed across conditions at each time point and a paired $t$-test was used to compare differences between pre and post. When one-way repeated measure ANOVAs were performed, a Fisher's least significant difference post $h o c$ test was used to determine where the differences were. Statistical significance was set at $p \leq 0.05$. 


\section{Results}

\section{Descriptive statistics}

All results are expressed as mean [95\% confidence interval (CI)] unless noted otherwise. The mean age, height, and body mass, respectively, were as follows: 24 (95\% CI: 22-26) years, 175 (95\% CI: 169-181) cm, and 83 (95\% CI: 75-92) kg. In addition, the average 1RM was 25.7 (95\% CI: 20.8-30.7) kg and the average arterial occlusion pressure was 151 (95\% CI: 138-164) $\mathrm{mmHg}$. This corresponded to an average applied pressure of 59 (95\% CI: 54-65) $\mathrm{mmHg}$ for the $40 \%$ arterial occlusion pressure conditions and 122 (95\% CI: 111-132) $\mathrm{mmHg}$ for the $80 \%$ arterial occlusion pressure conditions.

\section{$s E M G$}

There was an interaction for sEMG amplitude of the first 3 repetitions of each set $(p=0.003)$. sEMG amplitude was the highest during the 20\% $1 \mathrm{RM}$ condition and the first 3 repetitions of sets 2, 3, and 4 had significantly greater amplitude than the first 3 repetitions of set 1 (Table I). There was no interaction for sEMG during the final 3 repetitions of each set $(p=0.832)$; however, there was a main effect of condition $(p=0.002)$ with greater sEMG amplitude during the $20 \% 1 \mathrm{RM}$ conditions, and a main effect of time detailing higher sEMG in sets 2,3, and 4 when compared with set $1(p=0.019$; Table I).

\section{Repetitions}

There was an interaction for repetitions completed $(p<0.001)$ with the $20 \% 1 \mathrm{RM}$ conditions resulting in fewer repetitions during the final two sets (Table II), although post hoc comparisons were not statistically significant. Notably, the only conditions that did not result in all repetitions being completed were the $20 \% 1 \mathrm{RM}$ conditions.

\section{Acute muscle thickness}

For acute changes in muscle thickness (a marker of swelling), there was an interaction $(p<0.001)$ with all conditions increasing from pre to post (Table III). Acute muscle thickness appeared to be impacted by increasing pressures and loads, with the largest increase observed in the $20 \% 1 \mathrm{RM}$ and $80 \%$ arterial occlusion pressure condition.

\section{Torque}

There was an interaction $(p<0.001)$ for torque with both higher pressures and higher loads resulting in greater acute decrements in torque (a marker of fatigue; Table IV). Relative mean decrements in torque at the group level ranged from $7.5 \%$ in the $10 \% 1 \mathrm{RM} / 40 \%$ arterial occlusion to $44.5 \%$ in the $20 \% 1 \mathrm{RM} / 80 \%$ arterial occlusion condition.

\section{Discussion}

The main finding from this study was that the use of higher pressures during very low-load $(10 \%-20 \% 1 \mathrm{RM})$ exercise increased fatigue (decrement in torque) but did not increase sEMG amplitude. The only increase in sEMG amplitude occurred from increasing the exercise load independent of the pressures applied. Furthermore, we observed that the only conditions that did not complete all of the target repetitions were those performed using a $20 \% 1 \mathrm{RM}$ load. Finally, all conditions resulted in acute muscle swelling, with a slightly greater swelling response observed following higher pressures and loads. 
Table I. Surface electromyography (sEMG)

\begin{tabular}{|c|c|c|c|c|c|}
\hline & Set 1 & Set 2 & Set 3 & Set 4 & Time \\
\hline \multicolumn{6}{|l|}{ First 3 repetitions } \\
\hline $\begin{array}{l}10 \% 1 \mathrm{RM}, 40 \% \text { arterial } \\
\text { occlusion }\end{array}$ & $30(25)$ & $29(27)^{\mathrm{a}, \mathrm{b}, \mathrm{c}, \mathrm{d}}$ & $29(26)^{\mathrm{a}, \mathrm{b}}$ & $31(29)^{\mathrm{a}, \mathrm{b}}$ & ND \\
\hline $\begin{array}{l}10 \% 1 \mathrm{RM}, 80 \% \text { arterial } \\
\text { occlusion }\end{array}$ & $21(8)$ & $22(9)^{\mathrm{a}}$ & $25(14)^{\mathrm{a}}$ & $27(13)^{\mathrm{a}}$ & 1 vs. 3,$4 ; 2$ vs. 3,4 \\
\hline $\begin{array}{l}15 \% 1 \mathrm{RM}, 40 \% \text { arterial } \\
\text { occlusion }\end{array}$ & $24(11)$ & $27(7)^{b}$ & $28(10)^{\mathrm{a}}$ & $30(11)^{\mathrm{a}}$ & 4 vs. $1,2,3$ \\
\hline $\begin{array}{l}15 \% 1 \mathrm{RM}, 80 \% \text { arterial } \\
\text { occlusion }\end{array}$ & $24(6)$ & $28(7)^{\mathrm{b}, \mathrm{c}}$ & $30(9)^{a, b}$ & $31(9)^{\mathrm{a}}$ & 1 vs. $2,3,4 ; 2$ vs. 3 \\
\hline $\begin{array}{l}20 \% 1 \mathrm{RM}, 40 \% \text { arterial } \\
\text { occlusion }\end{array}$ & $32(14)$ & $35(9)^{\mathrm{d}}$ & $38(11)^{\mathrm{b}, \mathrm{c}}$ & $41(15)^{\mathrm{b}}$ & ND \\
\hline $\begin{array}{l}20 \% 1 \mathrm{RM}, 80 \% \text { arterial } \\
\text { occlusion }\end{array}$ & $29(10)$ & $39(13)^{d}$ & $44(16)^{c}$ & $44(15)^{\mathrm{b}}$ & 1 vs. $2,3,4$ \\
\hline \multicolumn{6}{|l|}{ Last 3 repetitions } \\
\hline $\begin{array}{l}10 \% 1 \mathrm{RM}, 40 \% \text { arterial } \\
\text { occlusion }^{\mathrm{a}}\end{array}$ & $37(28)$ & $37(31)$ & 37 (29) & $36(27)$ & 1 vs. $2,3,4$ \\
\hline $\begin{array}{l}10 \% \text { 1RM, } 80 \% \text { arterial } \\
\text { occlusion }^{\mathrm{a}}\end{array}$ & $30(12)$ & $34(16)$ & $37(20)$ & $36(16)$ & 1 vs. $2,3,4$ \\
\hline $\begin{array}{l}15 \% 1 \mathrm{RM}, 40 \% \text { arterial } \\
\text { occlusion }^{\mathrm{a}}\end{array}$ & $36(12)$ & 38 (13) & $40(12)$ & $40(13)$ & 1 vs. $2,3,4$ \\
\hline $\begin{array}{l}15 \% \text { 1RM, } 80 \% \text { arterial } \\
\text { occlusion }^{\mathrm{a}}\end{array}$ & $37(8)$ & $40(10)$ & $42(10)$ & $43(14)$ & 1 vs. $2,3,4$ \\
\hline $\begin{array}{l}20 \% 1 \mathrm{RM}, 40 \% \text { arterial } \\
\text { occlusion }^{\mathrm{b}}\end{array}$ & $53(16)$ & $56(18)$ & $57(21)$ & $55(21)$ & 1 vs. $2,3,4$ \\
\hline $\begin{array}{l}20 \% 1 \mathrm{RM}, 80 \% \text { arterial } \\
\text { occlusion }^{\mathrm{b}}\end{array}$ & $48(16)$ & $56(21)$ & $56(23)$ & $57(24)$ & 1 vs. $2,3,4$ \\
\hline
\end{tabular}

Based on the interaction term, simple effects were interpreted for the first 3 repetitions and main effects were interpreted for the last 3 repetitions. Superscript letters denote significant differences across condition within each set. If two conditions contain at least one of the same superscript letters, they are not statistically different from one another. The time column details significant differences within condition at each time point. All results are mean (standard deviation). 1RM: one-repetition maximum; ND: no significant differences

Contrary to our hypothesis, increasing the blood flow restriction pressure did not result in greater sEMG amplitude when lower loads were used. It should be mentioned that two individuals in this study had uncharacteristically high sEMG during the 10\%1RM conditions and this appeared to be the result of low SEMG amplitude during the preexercise MVC, which was used to normalize sEMG during exercise. The vastly differential response of these two individuals is apparent in the high standard deviations for the 10\% 1RM conditions (Table I), and this appeared to mask a more distinct gradient between sEMG and the load being used. Our sEMG measure was used as an indirect marker of motor unit recruitment, which is 
Table II. Repetitions

\begin{tabular}{|l|c|c|c|c|}
\hline & Set 1 & Set 2 & Set 3 & Set 4 \\
\hline $10 \% 1 \mathrm{RM}, 40 \%$ occlusion pressure & $30(0)$ & $15(0)$ & $15(0)$ & $15(0)$ \\
\hline $10 \% 1 \mathrm{RM}, 80 \%$ occlusion pressure & $30(0)$ & $15(0)$ & $15(0)$ & $15(0)$ \\
\hline $15 \% 1 \mathrm{RM}, 40 \%$ occlusion pressure & $30(0)$ & $15(0)$ & $15(0)$ & $15(0)$ \\
\hline $15 \% 1 \mathrm{RM}, 80 \%$ occlusion pressure & $30(0)$ & $15(0)$ & $15(0)$ & $15(0)$ \\
\hline $20 \% 1 \mathrm{RM}, 40 \%$ occlusion pressure & $30(0)$ & $15(0)$ & $15(0)$ & $14(1)$ \\
\hline $20 \% 1 \mathrm{RM}, 80 \%$ occlusion pressure & $30(0)$ & $15(0)$ & $13(2)$ & $12(4)$ \\
\hline
\end{tabular}

1RM: one-repetition maximum

Table III. Swelling

\begin{tabular}{|l|c|c|c|}
\hline & Pre & Post & $\Delta(\mathbf{9 5 \%}$ confidence interval) \\
\hline $10 \% 1 \mathrm{RM}, 40 \%$ occlusion pressure & $4.7(0.9)$ & $4.9(1.0)^{\mathrm{a}}$ & $0.1(0.1,0.2)^{*}$ \\
\hline $10 \% 1 \mathrm{RM}, 80 \%$ occlusion pressure & $4.7(1.0)$ & $5.0(0.9)^{\mathrm{a}, \mathrm{c}, \mathrm{d}}$ & $0.3(0.2,0.3)^{*}$ \\
\hline $15 \% 1 \mathrm{RM}, 40 \%$ occlusion pressure & $4.7(0.9)$ & $5.0(0.9)^{\mathrm{b}, \mathrm{e}}$ & $0.3(0.2,0.4)^{*}$ \\
\hline $15 \% 1 \mathrm{RM}, 80 \%$ occlusion pressure & $4.7(0.9)$ & $5.1(0.9)^{\mathrm{c}}$ & $0.4(0.3,0.4)^{*}$ \\
\hline $20 \% 1 \mathrm{RM}, 40 \%$ occlusion pressure & $4.7(0.9)$ & $5.1(1.0)^{\mathrm{a}, \mathrm{b}, \mathrm{c}, \mathrm{d}}$ & $0.3(0.2,0.4)^{*}$ \\
\hline $20 \% 1 \mathrm{RM}, 80 \%$ occlusion pressure & $4.7(0.9)$ & $5.1(0.9)^{\mathrm{a}, \mathrm{d}, \mathrm{e}}$ & $0.4(0.3,0.4)^{*}$ \\
\hline
\end{tabular}

Values are expressed in $\mathrm{cm}$ as the acute increase in muscle thickness measured immediately after the exercise protocol. Superscript letters indicate significant differences. If two conditions contain at least one of the same superscript letters, they are not statistically different from one another. All results are mean (standard deviation). 1RM: one-repetition maximum.

*Significant increase from pre to post within each condition

Table IV. Fatigue

\begin{tabular}{|c|c|c|c|}
\hline & Pre & Post & $\Delta(95 \%$ confidence interval $)$ \\
\hline $10 \% 1 \mathrm{RM}, 40 \%$ occlusion pressure & $68.9(27.8)$ & $63.7(28.8)^{\mathrm{a}}$ & $-5.2(-7.2,-3.2)^{*}$ \\
\hline $10 \% 1 \mathrm{RM}, 80 \%$ occlusion pressure & $72.5(27.3)$ & $55.5(19.2)^{\mathrm{a}}$ & $-17.0(-27.1,-6.9)^{*}$ \\
\hline $15 \% 1 \mathrm{RM}, 40 \%$ occlusion pressure & $72.2(27.5)$ & $57.1(21.3)^{\mathrm{a}}$ & $-15.0(-22.3,-7.9)^{*}$ \\
\hline $15 \% 1 \mathrm{RM}, 80 \%$ occlusion pressure & $68.6(29.0)$ & $47.8(22.9)^{\mathrm{b}}$ & $-20.7(-29.2,-12.2)^{*}$ \\
\hline $20 \% 1 \mathrm{RM}, 40 \%$ occlusion pressure & $70.3(27.6)$ & $45.9(21.5)^{\mathrm{b}, \mathrm{c}}$ & $-24.4(-32.9,-15.8)^{*}$ \\
\hline $20 \% 1 \mathrm{RM}, 80 \%$ occlusion pressure & $72.8(31.0)$ & $40.3(19.9)^{\mathrm{c}}$ & $-32.4(-44.8,-20.1)^{*}$ \\
\hline
\end{tabular}

Values are expressed in $\mathrm{Nm}$ as the drop in maximal isometric strength immediately after the exercise protocol. There was an interaction with different superscript letters indicating differences between groups at the post-measure. All results are mean (standard deviation). 1RM: one-repetition maximum.

*All groups decreased significantly from pre to post 
thought to be an important factor with respect to muscle growth (23). Importantly, sEMG is an estimate of the maximum motor unit recruitment at a given time and inferring muscle activation off of sEMG amplitude may be particularly problematic when using lower loads (10) as motor units can be derecruited and subsequently rerecruited to allow for the recovery of specific motor units $(27,28)$. Since there only needs to be a great enough force to overcome the load being lifted, it is possible to hypothesize that there were no true differences in motor unit recruitment, but rather, a greater number of motor units were required to be recruited at one specific time to overcome the $20 \%$ load as opposed to the $10 \%$ or $15 \%$ loads. Thus, we may have detailed the minimal sEMG amplitude required at one given time to overcome the specific load used, as opposed to identifying motor unit recruitment throughout the exercise protocol.

While we hypothesized that sEMG activity would increase across all loads and pressures as a result of a more stressful stimulus, the idea that sEMG did not increase with increasing pressures leads us to question whether this hypothesis was correct. We saw a clear gradient in the decrement in torque induced by each protocol, such that any increase in pressure and/or load resulted in greater fatigue (Table IV). Despite all protocols differing in our marker of fatigue, only the load had an impact on sEMG. Given that fatigue causes an increase in motor unit recruitment (3), it would seem as though the maximal sEMG that could be achieved within each given load was already met as it was not augmented by an increase in fatigue. The combined results of fatigue and sEMG appear to further detail the potential limitations of inferring motor unit recruitment from sEMG amplitude. A previous blood flow restriction study found that increasing the pressure from $40 \%$ to $80 \%$ of arterial occlusion pressure resulted in greater muscle growth when a 20\% 1RM load was used (17). We did not observe any changes in sEMG between the 20\% 1RM conditions under $40 \%$ and $80 \%$ arterial occlusion pressures, and would have inferred from our acute findings that both would have resulted in similar chronic muscle adaptations when in fact they differed. It should be mentioned that the previous training study incorporated lower-body exercise (17), whereas we examined upper-body exercise in this study. Even so, this discrepancy may further question how meaningful sEMG data is when attempting to infer chronic training adaptations based on acute resistance exercise protocols incorporating lower loads (10). Therefore, it remains unknown how important the pressure is with respect to very low-load blood flowrestricted exercise, but it appears that increasing the pressure results in decrements in torque (a marker of fatigue). Another previous study observed no differences in muscle growth in response to $40 \%$ and $90 \%$ arterial occlusion pressures when using a 30\% 1RM load (5), although it should be mentioned that these protocols did not differ in the acute decrements in torque (i.e., fatigue) caused by each protocol. This may support the hypothesis that the restrictive pressure may be more important as the relative load is decreased.

The increase in acute muscle thickness that was present across all conditions may demonstrate a similar anabolic potential, given the association between acute muscle swelling and muscle growth $(18,29)$. This hypothesis was made as concentric blood flow-restricted exercise induced greater acute swelling and long-term muscle growth when compared with eccentric blood flow restriction exercise (26), but this association with muscle growth may not be causative. Using muscle swelling as a marker of the anabolic potential of a given protocol should be interpreted with caution as simply applying the pressure cuff independent of exercise has been shown to induce muscle swelling (19), although this has been accompanied by an attenuation of atrophy but not an increase in muscle size (4). This may also detail that muscle swelling may be less sensitive to changes in pressure and loads in comparison with fatigue, 
given all protocols appeared to swell to a similar extent (Table III), yet clear differences in fatigue were observed (Table IV). While muscle swelling may be an important marker of muscle growth, it is unlikely to play a large mechanistic role in this process (20).

Finally, the reporting of repetitions demonstrates that only the $20 \% 1 \mathrm{RM}$ condition caused some individuals to reach volitional failure, with even fewer repetitions completed during the $20 \% 1 \mathrm{RM}$ condition combined with the higher pressure $(80 \%$ arterial occlusion pressure), as opposed to the $20 \% 1 \mathrm{RM}$ condition with the lower pressure $(40 \%$ arterial occlusion pressure). This would also support our hypothesis that perhaps higher pressures are more advantageous, given it brought individuals to, or closer to, volitional failure (23). Had this study been performed again, it may be beneficial to avoid using the traditional blood flow restriction protocol and simply have all individuals exercise to volitional failure as this would have ensured a common stimulus across all individuals (7). This would have allowed us to analyze the number of repetitions required to bring the individuals to volitional failure, which would also have detailed the importance of different pressure/load combinations. Thus, it may be more beneficial to steer away from the traditional blood flow restriction protocol assigning a set number of repetitions [ 1 set of 30 followed by 3 sets of 15 repetitions $(6,22)$ ] and simply administer protocols taking all individuals to volitional failure.

As with all observational studies, this study is not without limitations. We used a standard blood flow restriction protocol to help analyze the stimulus being applied within the literature, but this likely resulted in a differential stimulus across individuals depending on differences in repetitions that could be completed at a set load. In addition, we used sEMG as a marker of motor unit recruitment but did not analyze the activity of specific motor units; however, this method is commonly used to infer chronic muscle adaptations from acute protocols and was used to build a profile of each stimulus.

In conclusion, higher restriction pressures induce a greater level of fatigue but do not alter sEMG activity during low-load (10\%-20\% 1RM) blood flow-restricted exercise. These results may detail the limitation using sEMG to infer motor unit recruitment during low-load resistance training protocols, while also detailing that both higher pressures and loads contribute to the fatigue caused by blood flow-restricted exercise. Altogether, these results suggest that higher pressures may be more beneficial for blood flow-restricted exercise when very low loads are used; however, chronic training studies are necessary to clarify this hypothesis examining loads even lower than $20 \% 1 \mathrm{RM}$.

\section{Acknowledgements}

None.

\section{Conflict of interest}

The authors declare no conflict of interest.

\section{REFERENCES}

1. Abe T, Fujita S, Nakajima T, Sakamaki M, Ozaki H, Ogasawara R, Sugaya M, Kudo M, Kurano M, Yasuda T, Sato Y, Ohshima H, Mukai C, Ishii N: Effects of low-intensity cycle training with restricted leg blood flow on thigh muscle volume and $\mathrm{VO}_{2 \max }$ in young men. J. Sports Sci. Med. 9(3), 452-458 (2010)

2. Abe T, Kearns CF, Sato Y: Muscle size and strength are increased following walk training with restricted venous blood flow from the leg muscle, Kaatsu-walk training. J. Appl. Physiol. 100(5), 1460-1466 (2006) 
3. Adam A, De Luca CJ: Firing rates of motor units in human vastus lateralis muscle during fatiguing isometric contractions. J. Appl. Physiol. 99(1), 268-280 (2005)

4. Clark BC, Fernhall B, Ploutz-Snyder LL: Adaptations in human neuromuscular function following prolonged unweighting: I. Skeletal muscle contractile properties and applied ischemia efficacy. J. Appl. Physiol. 101(1), 256-263 (2006)

5. Counts BR, Dankel SJ, Barnett BE, Kim D, Mouser JG, Allen KM, Thiebaud RS, Abe T, Bemben MG, Loenneke JP: Influence of relative blood flow restriction pressure on muscle activation and muscle adaptation. Muscle Nerve 53(3), 438-445 (2016)

6. Dankel SJ, Jessee MB, Abe T, Loenneke JP: The effects of blood flow restriction on upper-body musculature located distal and proximal to applied pressure. Sports Med. 46(1), 23-33 (2015)

7. Dankel SJ, Jessee MB, Mattocks KT, Mouser JG, Counts BR, Buckner SL, Loenneke JP: Training to fatigue: the answer for standardization when assessing muscle hypertrophy? Sports Med. 47(6), 1021-1027 (2017)

8. Fahs CA, Loenneke JP, Thiebaud RS, Rossow LM, Kim D, Abe T, Beck TW, Feeback DL, Bemben DA, Bemben MG: Muscular adaptations to fatiguing exercise with and without blood flow restriction. Clin. Physiol. Funct. Imaging 35(3), 167-176 (2015)

9. Farup J, de Paoli F, Bjerg K, Riis S, Ringgard S, Vissing K: Blood flow restricted and traditional resistance training performed to fatigue produce equal muscle hypertrophy. Scand J. Med. Sci. Sports 25(6), 754-763 (2015)

10. Fisher J, Steele J, Smith D: High- and low-load resistance training: interpretation and practical application of current research findings. Sports Med. 47, 393-400 (2017)

11. Head P, Austen B, Browne D, Campkin T, Barcellona M: Effect of practical blood flow restriction training during bodyweight exercise on muscular strength, hypertrophy and function in adults: a randomised controlled trial. Int. J. Ther. Rehabil. 22(6), 263-271 (2015)

12. Hermens HJ, Freriks B, Disselhorst-Klug C, Rau G: Development of recommendations for SEMG sensors and sensor placement procedures. J. Electromyogr. Kinesiol. 10(5), 361-374 (2000)

13. Järvholm U, Styf J, Suurkula M, Herberts P: Intramuscular pressure and muscle blood flow in supraspinatus. Eur. J. Appl. Physiol. 58(3), 219-224 (1988)

14. Kacin A, Strazar K: Frequent low-load ischemic resistance exercise to failure enhances muscle oxygen delivery and endurance capacity. Scand J. Med. Sci. Sports 21(6), e231-e241 (2011)

15. Kang DY, Kim HS, Lee KS, Kim YM: The effects of bodyweight-based exercise with blood flow restriction on isokinetic knee muscular function and thigh circumference in college students. J. Phys. Ther. Sci. 27(9), 27092712 (2015)

16. Laurentino G, Ugrinowitsch C, Aihara AY, Fernandes AR, Parcell AC, Ricard M, Tricoli V: Effects of strength training and vascular occlusion. Int. J. Sports Med. 29(8), 664-667 (2008)

17. Lixandrão ME, Ugrinowitsch C, Laurentino G, Libardi CA, Aihara AY, Cardoso FN, Tricoli V, Roschel H: Effects of exercise intensity and occlusion pressure after 12 weeks of resistance training with blood-flow restriction. Eur. J. Appl. Physiol. 115(12), 2471-2480 (2015)

18. Loenneke JP, Fahs CA, Rossow LM, Abe T, Bemben MG: The anabolic benefits of venous blood flow restriction training may be induced by muscle cell swelling. Med. Hypotheses 78(1), 151-154 (2012)

19. Loenneke JP, Fahs CA, Thiebaud RS, Rossow LM, Abe T, Ye X, Kim D, Bemben MG: The acute muscle swelling effects of blood flow restriction. Acta Physiol. Hung. 99(4), 400-410 (2012)

20. Loenneke JP, Kim D, Fahs CA, Thiebaud RS, Abe T, Larson RD, Bemben DA, Bemben MG: Effects of exercise with and without different degrees of blood flow restriction on torque and muscle activation. Muscle Nerve 51(5), 713-721 (2015)

21. Loenneke JP, Wilson GJ, Wilson JM: A mechanistic approach to blood flow occlusion. Int. J. Sports Med. 31(1), 1-4 (2010)

22. Loenneke JP, Wilson JM, Marín PJ, Zourdos MC, Bemben MG: Low intensity blood flow restriction training: a meta-analysis. Eur. J. Appl. Physiol. 112(5), 1849-1859 (2011)

23. Morton RW, McGlory C, Phillips SM: Nutritional interventions to augment resistance training-induced skeletal muscle hypertrophy. Front. Physiol. 6, 245 (2015)

24. Motykie GD, Zebala LP, Caprini JA, Lee CE, Arcelus JI, Reyna JJ, Cohen EB: A guide to venous thromboembolism risk factor assessment. J. Thromb. Thrombolysis 9(3), 253-262 (2000)

25. Pearson SJ, Hussain SR: A review on the mechanisms of blood-flow restriction resistance training-induced muscle hypertrophy. Sports Med. 45(2), 187-200 (2015)

26. Weir JP: Quantifying test-retest reliability using the intraclass correlation coefficient and the SEM. J. Strength Cond. Res. 19(1), 231-240 (2005) 
27. Westad C, Westgaard RH, De Luca CJ: Motor unit recruitment and derecruitment induced by brief increase in contraction amplitude of the human trapezius muscle. J. Physiol. 552(Pt. 2), 645-656 (2003)

28. Westgaard RH, de Luca CJ: Motor unit substitution in long-duration contractions of the human trapezius muscle. J. Neurophysiol. 82(1), 501-504 (1999)

29. Yasuda T, Loenneke JP, Thiebaud RS, Abe T: Effects of blood flow restricted low-intensity concentric or eccentric training on muscle size and strength. PLoS One 7(12), e52843 (2012) 